\title{
Resolution of photonic crystal superprism
}

\author{
Toshihiko Baba ${ }^{\text {a) }}$ and Takashi Matsumoto \\ Department of Electrical and Computer Engineering, Yokohama National University, 79-5 Tokiwadai, \\ Hodogayaku, Yokohama 240-8501, Japan
}

(Received 3 June 2002; accepted for publication 29 July 2002)

\begin{abstract}
We theoretically investigated the performance of the photonic crystal superprism, that is, the propagating beam quality, the wavelength sensitivity, and the resolution as a narrow band filter at 1.5- $\mu \mathrm{m}$-wavelength range. First, we defined the equi-incident-angle curve in the Brillouin zone. Next, we mapped three parameters that represented the abovementioned performance over the Brillouin zone. As a result, we found a narrow design window that allows a high resolution of 0.4 $\mathrm{nm}$ along an equi-incident-angle curve but requires an incident beam width of over $100 \mu \mathrm{m}$ and a device length of centimeter order. It can be an essential high efficiency filter if the input end of the crystal is optimized and the propagation loss is suppressed. (c) 2002 American Institute of Physics.

[DOI: 10.1063/1.1508813]
\end{abstract}

The superprism is a function of a photonic crystal (PC), which allows wide-angle deflection of a light beam by a slight change of wavelength and incident angle. Such an effect occurs due to the peculiar dispersion characteristics in the frequency range higher than the photonic band gap, as theoretically discussed and experimentally demonstrated by Russell, ${ }^{1}$ Kosaka et al., ${ }^{2}$ Chigrin et al. ${ }^{3}$ and others. The most fundamental application of the superprism is a diffractiontype narrow band wavelength filter, which is used by fixing the incident angle of the light beam. It can be further applied to a dispersion compensation device ${ }^{4}$ and to a light deflection device. ${ }^{5}$ Up to now, however, there are no reports on the quantitative estimation of the wavelength resolution, the most essential performance of these devices. Generally, the superprism simultaneously exhibits a high wavelength sensitivity and a wide angle divergence of light beam when the dispersion characteristic changes abruptly (e.g., at the band edge). Therefore, the condition for the maximum resolution is not given simply by the condition for the highest wavelength sensitivity. ${ }^{2}$ In this study, we considered these characteristics and theoretically estimated the resolution of the superprism by analyzing dispersion characteristics of some sample structures.

As shown in Fig. 1(a), we consider the situation in which a Gaussian beam of a finite width is incident to a twodimensional (2-D) PC and is deflected inside the PC. The deflection angle can be analyzed from an equifrequency curve of a dispersion surface in the Brillouin zone. The wave vector in the $\mathrm{PC}, \mathbf{k}_{\mathrm{c}}$, is determined so that the tangential component of the incident wave vector $\mathbf{k}_{\text {in }}$ against the input end of the PC is conserved. The direction of propagation is determined by the gradient of the dispersion surface at crosspoint $\mathrm{A}$ of the equifrequency curve and the construction line. Now, we introduce the equi-incident-angle curve for a better understanding of the superprism. It is a locus of point A in the Brillouin zone against a constant incident angle and varying frequency of light. Fig. 1(b) shows the schematic of the second photonic band. Equi-incident-angle curves become

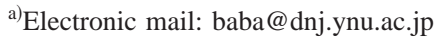

like vertical lines for this band. If one aims at a wavelength filter used for a certain incident angle of a light beam, one has to know the dispersion characteristic along the corresponding equi-incident-angle curve.

A Gaussian beam of a finite width can be expanded by plane waves with a Gaussian spectrum of the incident angle. The full spectral width at $1 / e^{2}$ intensity is given as $2 \theta_{\mathrm{D}}$ $=\lambda / \pi n w_{0}$, where $\lambda$ is the wavelength in vacuum, $n$ is the refractive index of the incident medium and $2 w_{0}$ is the spot diameter at $1 / e^{2}$ intensity. For example, $2 w_{0}=120 \mu \mathrm{m}$ corresponds to $2 \theta_{\mathrm{D}}=0.3^{\circ}$, when $\lambda=1.55 \mu \mathrm{m}$ and $n=3.065$. On the other hand, the light propagating in the PC is no longer a Gaussian beam, since each plane wave component is expanded into Bloch waves at the input end of the PC and is affected by the complex dispersion characteristic. However, the envelope of the light intensity profile can be approximated by a Gaussian when $2 \theta_{\mathrm{D}}$ is sufficiently small. Then, the beam width $2 w$ in the $\mathrm{PC}$ at a far distance from the input end is expressed as

$$
2 w \cong 2 \frac{\lambda L}{\pi n w_{0}} p,
$$

where $p \equiv\left(\partial \theta_{\mathrm{c}} / \partial \theta_{\mathrm{i}}\right)$ for the incident beam angle $\theta_{\mathrm{i}}$ and the propagating beam angle in the $\mathrm{PC}$ is $\theta_{\mathrm{c}}$. This parameter

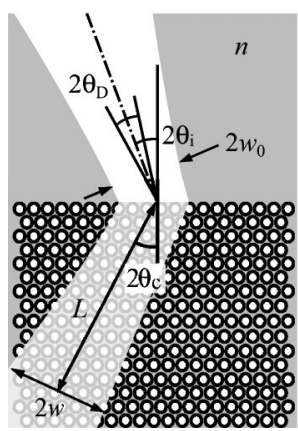

(a)

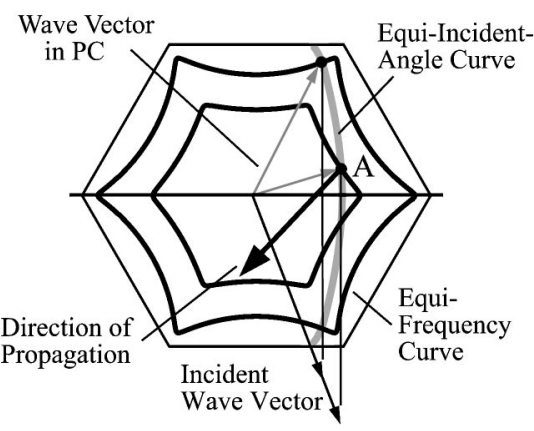

(b)
FIG. 1. Correspondence of real space and wave number space. (a) Deflection of Gaussian beam incident to 2-D PC. (b) Schematic of equifrequency curves (solid curves) and equi-incident-angle curves (gray curves) in the Brillouin zone. 

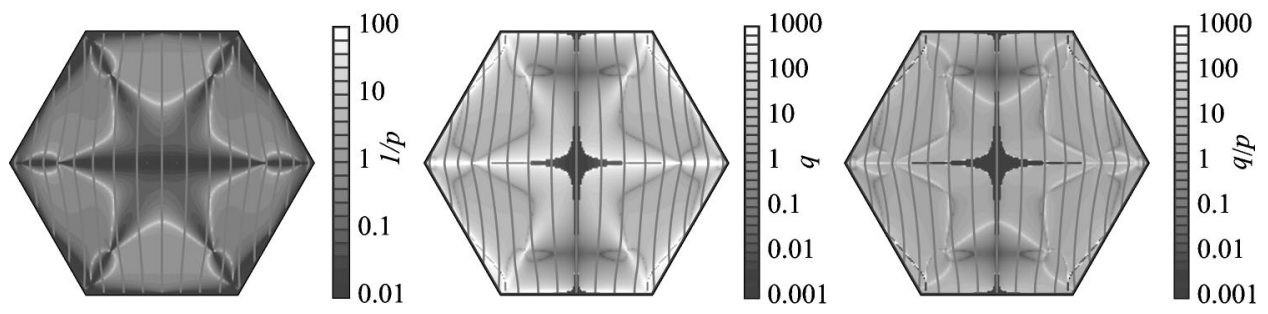

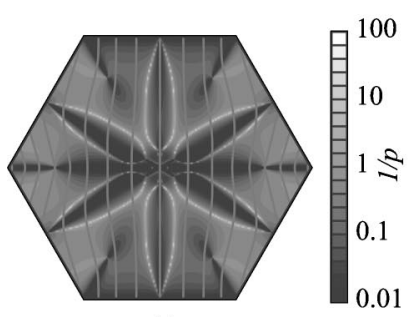

(a)

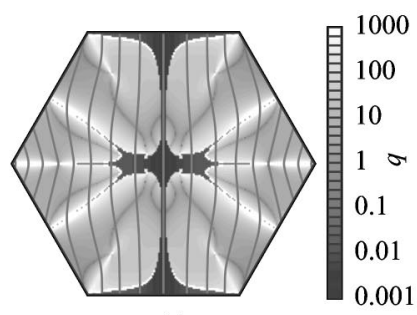

(b)

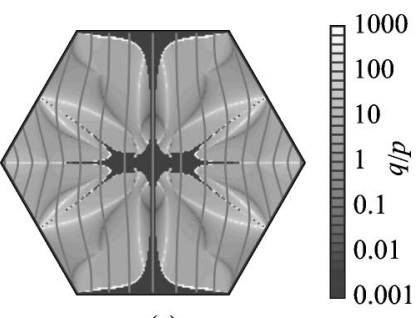

(c)
FIG. 2. Shaded drawing of (a) beam collimation parameter $1 / p$, (b) angular dispersion parameter $q$, and (c) resolution parameter $q / p$ in 2-D PC of triangular lattice airholes. Top and bottom denote the second and the third bands, respectively. represents the degree of beam divergence in the PC. (Therefore, $1 / p$ is a parameter for the beam collimation.) In Eq. (1), $L$ is the distance from the input end, which satisfies

$$
L>\frac{\pi n w_{0}^{2}}{\lambda} \frac{1}{p} \text {. }
$$

Since the beam width of Eq. (1) occupies the far field angle $2 w / L$, the wavelength resolution is given by

$$
\frac{\Delta \lambda}{\lambda}=\frac{2 w / L}{a / \lambda} \frac{\partial(a / \lambda)}{\partial \theta_{c}} \cong \frac{2 \lambda^{2}}{\pi n w_{0} a}\left(\frac{q}{p}\right)^{-1},
$$

where $a$ is the lattice pitch of the PC, and $q \equiv \partial \theta_{\mathrm{c}} / \partial(a / \lambda)$. This parameter represents the angular dispersion (wavelength sensitivity) of the PC. When $\lambda=1.55 \mu \mathrm{m}, 2 w_{0}=120 \mu \mathrm{m}$, and $n=3.065$, a wavelength resolution of $0.4 \mathrm{~nm}$ is obtained by $q / p>75$. In the following, we call $q / p$ the resolution parameter.

We investigated parameters $1 / p, q$, and $q / p$ by calculating dispersion surfaces of a typical PC, that is, the 2-D PC of triangular lattice airholes. We assumed the background refractive index to be equal to that of the incident medium, 3.065 , the index of the airholes to be 1.0, and the normalized diameter of the airholes $2 r / a=0.624$. Dispersion surfaces were obtained by calculating eigenfrequencies over the Brillouin zone using Ho's plane wave expansion method. ${ }^{6} \mathrm{We}$ limited the number of plane waves to 37 , since differential calculations of $1 / p$ and $q$ require a large computational task to obtain sufficient accuracy even when the dispersion surface exhibits abrupt changes. This number is not sufficient for the convergence of results, but it provides the outline of the parameters within $1 \%$ error for lower order photonic bands, which are of interest in this study. Figure 2 shows results for the second and the third bands. Overall, $1 / p$ is small and $q$ is large in the Brillouin zone. This means that the light beam is easily diverged and its deflection is sensitive to the wavelength. Both of them are remarkable at abruptly changing dispersion surfaces. Under such a condition, the resolution parameter $q / p$ becomes rather small, since the large $q$ is compensated by the small $1 / p$. Close to this condition, however, there exist narrow regions that satisfy $q / p>75$. Fortunately, some of these regions lie along the equi-incident-angle curve. This means that high resolution is expected in a wide spectral range against a fixed incident beam angle.

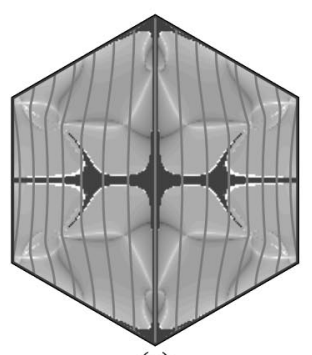

(a)

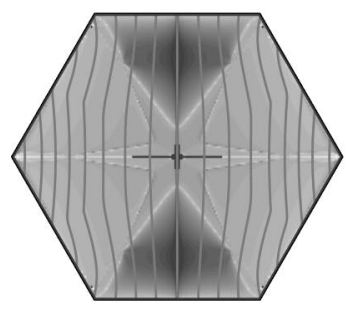

(d)

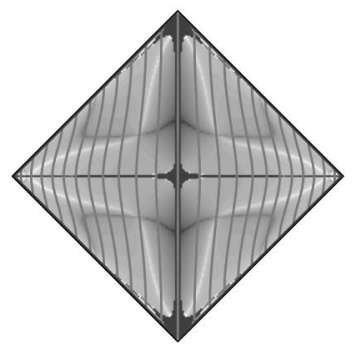

(b)

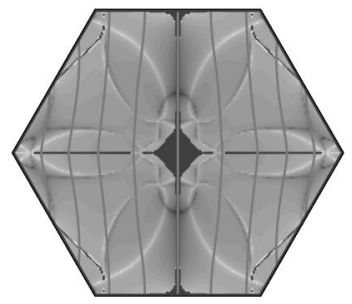

(e)

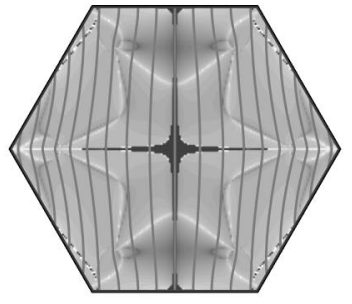

(c)

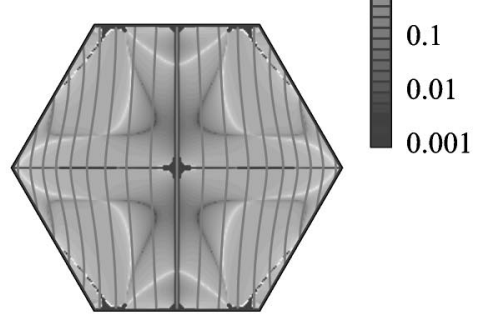

(f)
FIG. 3. Dependence of resolution parameter $q / p$ on structural details of the PC. (a) Triangular lattice rotated by $30^{\circ}$. (b) Square lattice rotated by $45^{\circ}$. (c) High background index of 6.0. (d) Larger normalized diameter of airholes $2 r / a=0.9$. (e) Smaller normalized diameter of airholes $2 r / a=0.2$. (f) Elliptical airholes of laterally $0.9 a$ $\times$ vertically $0.4 a$. 
TABLE I. Relation of beam width, device length and number of resolution in superprism wavelength filter. Target wavelength resolutions are (a) 0.4 $\mathrm{nm}$ and (b) $1.0 \mathrm{~nm}$.

\begin{tabular}{|c|c|c|c|c|}
\hline & & $2 w_{0}$ & $L$ & $M$ \\
\hline \multirow[t]{3}{*}{ (a) } & Rotated square lattice & $140 \mu \mathrm{m}$ & $19 \mathrm{~cm}$ & 51 \\
\hline & High index background & $75 \mu \mathrm{m}$ & $7 \mathrm{~cm}$ & 44 \\
\hline & Elliptical airholes & $115 \mu \mathrm{m}$ & $13 \mathrm{~cm}$ & 56 \\
\hline \multirow[t]{3}{*}{ (b) } & Rotated square lattice & $85 \mu \mathrm{m}$ & $4 \mathrm{~cm}$ & 92 \\
\hline & High index background & $48 \mu \mathrm{m}$ & $2 \mathrm{~cm}$ & 22 \\
\hline & Elliptical airholes & $70 \mu \mathrm{m}$ & $4 \mathrm{~cm}$ & 86 \\
\hline
\end{tabular}

Figure 3 shows the dependence of the resolution parameter $q / p$ on structural details. The optimum region is influenced by the lattice, its rotation, the background index, and the shape of the airholes. In addition, for the nonrealistic background index of 6.0, the deformation of airholes into an elliptical shape of laterally $0.9 a \times$ vertically $0.4 a$ is effective for the expansion of this region. The width of this region restricts the angular spectral width $2 \theta_{\mathrm{D}}$ of the incident beam and hence the spatial width of the incident beam. On the other hand, the length of this region along the equi-incidentangle curve determines the number of resolution points $M$. They are estimated with the required device length for each structure, as summarized in Table I. If the target resolution is $0.4 \mathrm{~nm}$, the incident beam width and the device length must be over $100 \mu \mathrm{m}$ and over $10 \mathrm{~cm}$, respectively. For a lower resolution, these requirements are relaxed. The device length can be further shortened to less than half by employing a multi-reflection-type structure with the zigzag optical path. As a result, the size and the performance of the superprism are almost comparable to those of a silica-based arrayed waveguide grating (AWG) filter.

Here, let us consider whether the essential resolution of the superprism is high or low. It is easy to compare the resolution parameter of the superprism with that of the standard 1-D grating with the first diffraction order. A simple calculation indicates that $q / p>75$ is achieved in the grating only when $\theta_{\mathrm{i}}>87.2^{\circ}$. Such oblique incidence of a light beam needs a very large grating and an extraordinary long distance of beam propagation in the free space to resolve the wavelength without using a focusing system. Thus, we can conclude that the resolution of the superprism is essentially high. However, as shown in Table I, the device size is larger (or in other words, the resolution is lower) than expected at the first stage of the study on the superprism. ${ }^{2}$ The reason considered is that only lower order photonic bands are usable in the superprism; the second and the third bands correspond to the first order diffraction in the standard grating. At higher bands than the fourth, a high diffraction efficiency toward unique direction cannot be expected, since some multiple bands appear at the same frequency and the branching of the propagating beam occurs. ${ }^{7}$ Therefore, it is difficult to gain resolution by increasing the order of diffraction to match those in a grating or in an AWG. One promising advantage of the superprism is the possibility of a high diffraction efficiency, which owes to the use of lower order bands. If the efficient mode matching is achieved at the input end of the PC, as described in Ref. 8, the superprism will be a filter with $100 \%$ efficiency.

In conclusion, we have discussed the theory of the performance of the superprism. The dispersion surface analysis indicated that the triangular lattice 2-D PC of elliptical airholes can be a wavelength filter with a resolution of $0.4 \mathrm{~nm}$, a resolution number of 56 , and a device size of $(6.5 \mathrm{~cm})^{2}$, which are comparable to those of a silica-based AWG filter. The diffraction efficiency is potentially high in the superprism due to its low diffraction order. If the slab structure is used as a 2-D PC, careful consideration for the light cone problem $^{9}$ is necessary to reduce the radiation loss. If the light is incident to the PC from the butt-joined optical fiber, a beam expander must be integrated. These are other issues to be managed for practical use.

The authors would like to thank Prof. Y. Kokubun, Yokohama National University, and Prof. K. Iga and Prof. F. Koyama, Tokyo Institute of Technology, for valuable suggestions. This work was partly supported by The Grant-in-Aid \#10210203 by the Ministry of Education, Science, Sports and Culture, and by CREST \#530-13 of the Japan Science and Technology Corporation.

${ }^{1}$ P. St. J. Russell and T. B. Birks, Photonic Band Gap Materials, edited by C. M. Soukoulis, (Kluwer, Dordrecht, 1996), p. 71.

${ }^{2}$ H. Kosaka, T. Kawashima, A. Tomita, M. Notomi, T. Tamamura, T. Sato, and S. Kawakami, Phys. Rev. B 58, 10096 (1998).

${ }^{3}$ D. N. Chigrin and C. M. S. Torres, Photonic Crystals and Light Localization in the 21st Century (Kluwer, Dordrecht, 2001), p. 173.

${ }^{4}$ H. Kosaka, T. Kawashima, A. Tomita, M. Notomi, T. Tamamura, T. Sato, and S. Kawakami, Appl. Phys. Lett. 74, 1370 (1999).

${ }^{5}$ T. Baba and M. Nakamura, IEEE J. Quantum Electron. 38, 909 (2002).

${ }^{6}$ K. M. Ho, C. T. Chan, and C. M. Soukoulis, Phys. Rev. Lett. 65, 3152 (1990).

${ }^{7}$ H. Kosaka, T. Kawashima, A. Tomita, M. Notomi, T. Tamamura, T. Sato, and S. Kawakami, Appl. Phys. Lett. 74, 1212 (2001).

${ }^{8}$ T. Baba and D. Ohsaki, Jpn. J. Appl. Phys. 40, 5920 (2001)

${ }^{9}$ T. Ochiai and K. Sakoda, Phys. Rev. B 63, 125107 (2001). 